\title{
Computed tomographic anatomy of hepatic artery in normal beagle dogs
}

\author{
Soochan Kim ${ }^{1}$, Seongmok Jeong ${ }^{1}$, Heechun Lee ${ }^{2}$, Youngwon Lee ${ }^{1}$, Hojung Choi ${ }^{1, *}$ \\ ${ }^{I}$ College of Veterinary Medicine and Research Institute of Veterinary Medicine, Chungnam National University, Daejeon 34134, Korea \\ ${ }^{2}$ College of Veterinary Medicine and Research Institute of Life Sciences, Gyeongsang National University, Jinju 52828, Korea
}

\begin{abstract}
This study was performed to examine the visualization and anatomical variants of the hepatic artery with dual-phase computed tomography (CT) angiography and three-dimensional volume rendering imaging analysis in clinically normal dogs. Seven healthy beagle dogs were enrolled and underwent dual CT angiography. Arterial phase images could be obtained with multi-detector CT angiography using the fixed-scan method in these dogs. Contrast enhancement of the hepatic parenchyma was quite minimal because of the unique blood supply system of the liver. In most dogs, the main hepatic arterial branches were the right lateral branch, left branch, and right medial branch. Although hepatic arterial variation appears to be common in dogs, only one dog in this study had the caudate lobar branch as the first branch of the hepatic artery. Further study on a larger number of dogs with CT images will be needed to identify and classify the pattern of hepatic arterial variations.
\end{abstract}

Keywords: hepatic artery, dog, computed tomography

*Corresponding author

Hojung Choi

College of Veterinary Medicine and

Research Institute of Veterinary Medicine, Chungnam National University, 99 Daehak-ro, Yuseong-gu, Daejeon 34134, Korea

Tel: +82-42-821-6770

Fax: +82-42-821-6703

E-mail: hjchoi@cnu.ac.kr

ORCID:

Soochan Kim

https://orcid.org/0000-0003-3457-5964

Seongmok Jeong

https://orcid.org/0000-0001-8653-5927

Youngwon Lee

https://orcid.org/0000-0003-3207-0989

Heechun Lee

https://orcid.org/0000-0001-5936-9118

Hojung Choi

https://orcid.org/0000-0001-7167-0755

Conflict of Interest

There is no conflict of interest.

Received: January 16, 2019

Revised: May 8, 2019

Accepted: May 22, 2019

\section{Introduction}

The anatomic variations of the hepatic arteries have been studied well and classified into various types in human medicine [1]. Knowledge of the hepatic artery variants is very important when planning vascular procedures, infusion therapy, or transarterial chemo-embolization of liver neoplasms, and liver transplantation [2]. The multi-detected computed tomography (CT) angiography is believed to be the gold standard for preoperative imaging evaluation method of the hepatic vasculature.

In the anatomic literature for dogs, the celiac artery is the first visceral branch from the ventral surface of the abdominal aorta. The hepatic artery runs cranioventrally and to the right, in a groove of the pancreas. At the level of the porta hepatis, the hepatic artery sends three to five branches into each liver lobe [3]. After giving off its hepatic branches, the hepatic artery turns caudally in the lesser omentum, and forms the small right gastric and a much larger gastroduodenal artery at the region of the pylorus [3]. Schmidt et al. [4] studied the branching patterns of the hepatic artery and found three major types in 51 dogs: a single hepatic artery trunk; two separate branches of the hepatic artery; and three, four, or five branches that originated directly from the hepatic artery. In a more recent study, Ursic et al. [5] examined the portal vein and hepatic artery ramifications with liver corrosion casts in 20 dogs. They found that the right lateral, right medial, and left branches are the major arteries originating from the hepatic artery, but their origin and course vary among individual dogs [5].

In veterinary medicine, there are some reports describing the hepatic arterial ramifications using CT angiography [6,7]. They agreed that the hepatic vein and portal vein have a consistent pattern of ramification. On the other hand, there were some discrepancies for the hepatic artery branching pattern in previous imaging studies with anatomic corrosion cast studies in dogs. This study visualized and estimated the anatomical variants of the hepatic artery with dual-phase CT angiography and 3-dimensional (3D) volume rendering imaging analysis in clinically normal dogs. 
Soochan Kim, Seongmok Jeong, Heechun Lee, Youngwon Lee, Hojung Choi

\section{Materials and Methods}

\section{Experimental animals}

This study was performed under the guidance of the Chungnam National University Animal Care and Use Committee. This study was a prospective and anatomic study design. Seven clinically normal beagle dogs (intact males) with an age of 4 to 6 years (mean age: 5.14 years) and weighing 8 to $13.7 \mathrm{~kg}$ were used. The dogs did not show any clinical signs of any liver diseases. Physical examinations, complete blood cell counts, serum chemistry analyses, thoracic and abdominal radiography, and sonography were performed to confirm that the dogs were clinically healthy.

\section{CT scan technique}

Prior to the CT scan, the dogs were fasted for $12 \mathrm{~h}$, but given access to water ad libitum. Anesthesia was induced with $3.0 \mathrm{mg} / \mathrm{kg}$ of alfaxalone (Alfaxan ${ }^{\circledR}$ inj., Jurox Pharm. Co. Ltd., Australia) intravenously. After endotracheal intubation, anesthesia was maintained with isoflurane (Ifran ${ }^{\circledR}$, Hana Pharm. Co. Ltd., Korea) and oxygen. The anesthetized dogs were placed in sternal recumbency on the CT table, and a 20gauge over-the-needle catheter in the cephalic vein was connected to an angiographic power injector (Salient ${ }^{\mathrm{TM}}$, Imaxeon Pty. Ltd., Australia). To avoid respiratory motion artifacts, each dog was hyperventilated manually before the CT scan, and the positive pressure ventilation with breath-holding technique $\left(10 \mathrm{~cm} \mathrm{H}_{2} \mathrm{O}\right)$ was applied during scanning. A 32detector-row CT scanner (Alexion ${ }^{\mathrm{TM}}$, Toshiba, Japan) was used with the following parameters: $120 \mathrm{kVp}$ and $150 \mathrm{~mA}$ settings, a scan speed of 0.75 -sec rotation, 1-mm slice thickness, and 1.438 collimation beam pitch. A survey helical CT scan was obtained from the cranial most aspect of the diaphragm to the caudal aspect of the third lumbar vertebra in the cranial-to-caudal direction. A dual-phase angiographic scan was performed using a fixed-scan method after the survey scan. CT contrast medium (Omnipaque ${ }^{\circledR}$, GE healthcare, Ireland, Ireland) with a dose of $600 \mathrm{mg}$ iodine $/ \mathrm{kg}$ and an injection rate of $4 \mathrm{~mL} / \mathrm{sec}$ was injected through a 20 -gauge IV catheter in the cephalic vein. Arterial phase scan was started at 6 to $7 \mathrm{sec}$ after injection in the caudal-to-cranial direction. In addition, portal phase images were obtained from scanning at 29-32 sec after the start of contrast media injection in the cranial-to-caudal direction.

\section{Image analyses}

The acquired CT images were evaluated using a commercial software program (Xelis ${ }^{\circledR}$, Infinitt Healthcare Co. LTD., Korea). Arterial ramifications were recorded from the celiac artery to the hepatic arterial branches. The vessel diameters were recorded for two arteries: celiac artery and hepatic artery, which have a consistent anatomic location and size. Their sizes were measured three to five times and the mean values were used as data. Attenuation values (Hounsfield unit; HU) of the hepatic parenchyma were measured on the dual phase images. The circular region of interest (ROI) with a mean area of $30 \mathrm{~mm}^{2}$ were placed on the hepatic parenchyma at the image of the 11th thoracic vertebra level avoiding hepatic vasculatures. The attenuation values were measured five times at different sites and the mean values were used as data. A $3 \mathrm{D}$ reconstruction of the hepatic artery was made using volume rendering software. The resulting 3D images of the cranial abdominal arteries were manipulated using rotation, cut planes, and controlling of the brightness and opacity to demonstrate the hepatic branches. The hepatic artery and branches were reviewed carefully and compared with transverse images to identify them and avoid deleting of any small branches.

\section{Results}

No abnormalities in hepatic parenchyma, biliary system and hepatic vasculature were observed on the survey CT images. Arterial phase images were obtained successfully without contrast media contamination into the portal vein. Minimal opacification of the hepatic parenchyma was observed grossly. On the portal phase images, the branches of the hepatic artery were not observed anymore, and the hepatic parenchyma was diffusely enhanced compared to the survey and arterial phase images (Fig. 1). These results were con-
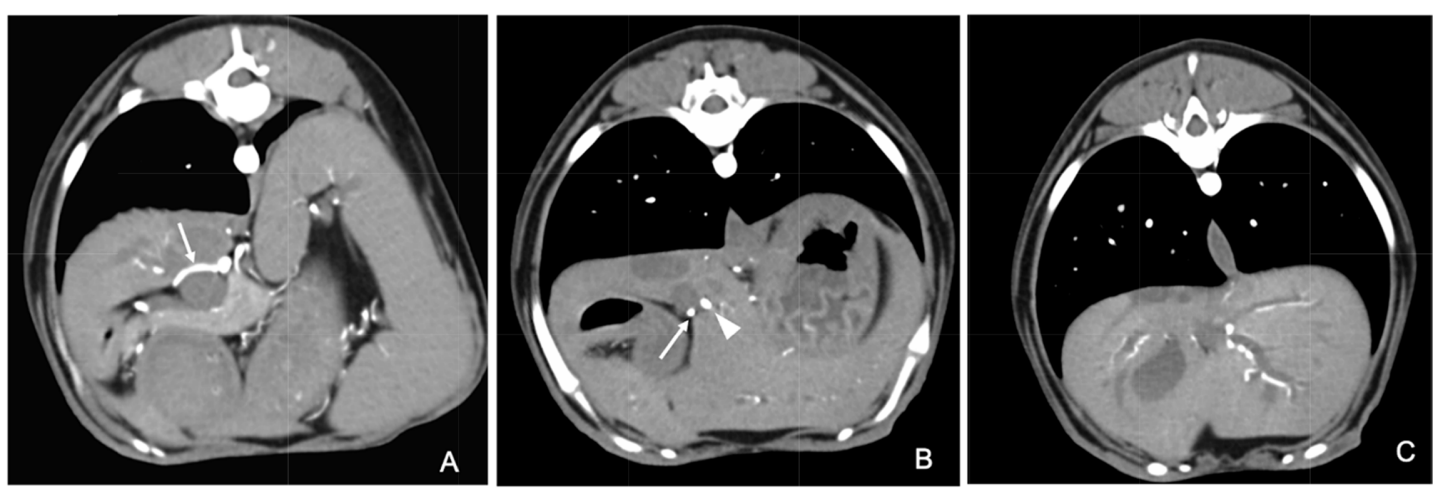

Fig. 1. The first branch from hepatic artery was mostly right lateral branch (arrow) seen dorsal aspect of main portal vein at the porta hepatis level (A). More cranially, the left branch (arrowhead) and right medial branch (arrow) were branched (B) and supplies each lobes (C). 


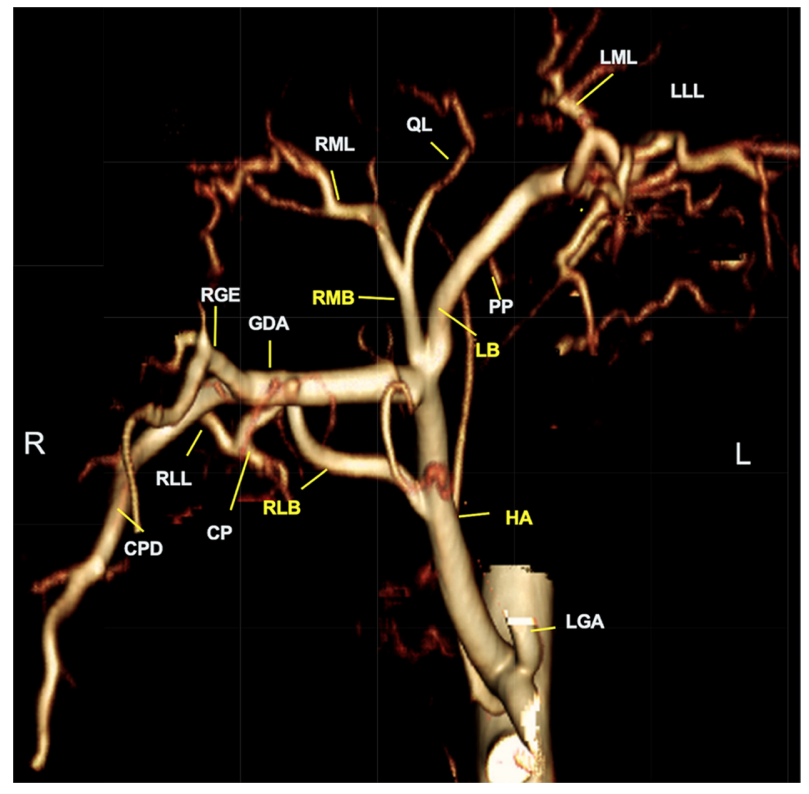

Fig. 2. Ventro-dorsal arterial phase $3 \mathrm{D}$ volume rendering image of a normal dog. The left gastric artery (LGA) and splenic artery were removed from the images for better visualization of hepatic arterial branches. Hepatic artery (HA) branched first right lateral branch (RLB), and relatively large left branch (LB) and small right medial branch (RMB). GDA, gastroduodenal artery; $\mathrm{CP}$, caudate process branch; $\mathrm{PP}$, papillary process branch; RGE, right gastroepiploic artery; CPD, cranial pancreatic duodenal artery; RLL, right lateral lobe; RML, right medial lobe; QL, quadrate lobe; LLL, left lateral lobe; LML, left medial lobe artery.
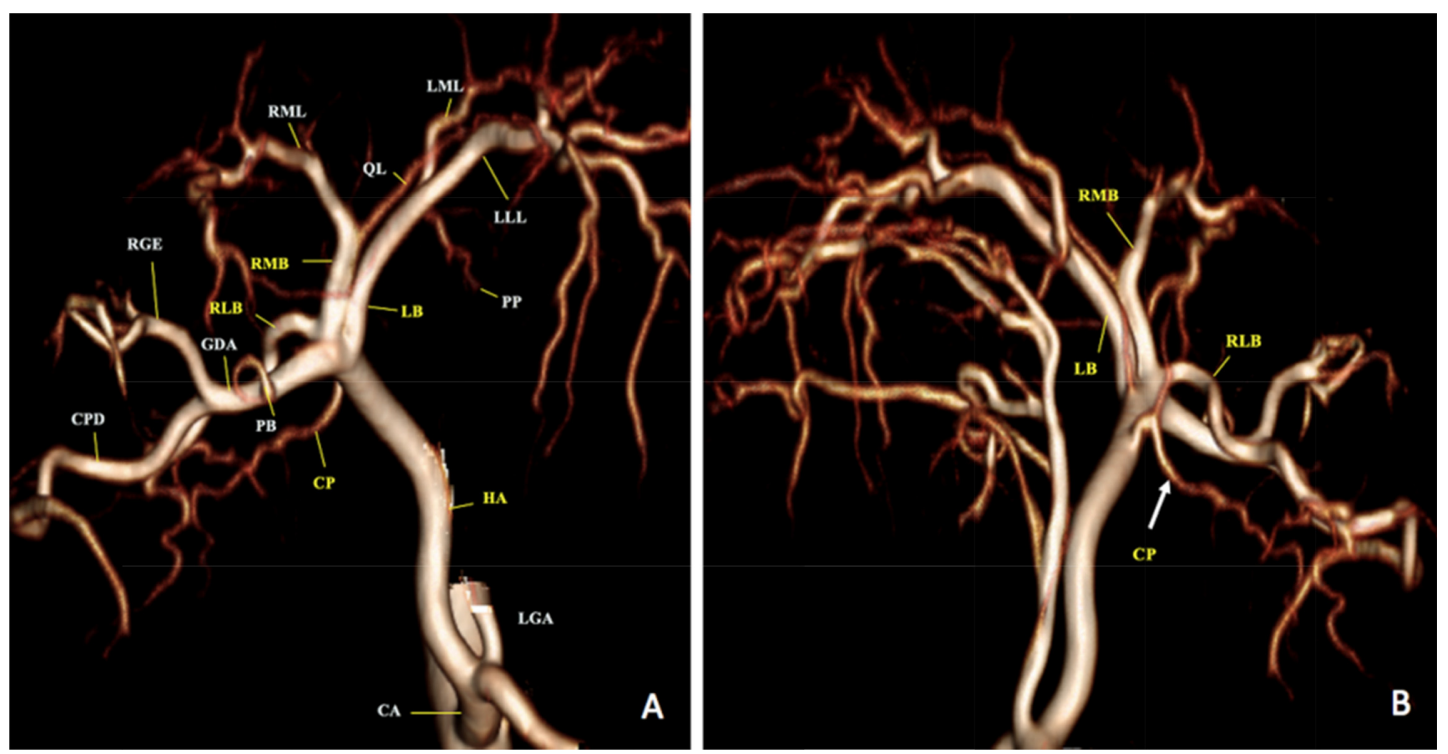

Fig. 3. Only one dog had a variant hepatic artery ramification compared to other dogs. Caudate process artery arose directly from the hepatic artery, which was the first and the most dorsal branch (A and B). Hepatic artery was subsequently divided into the left, right medial, and right lateral branches almost at the same time. GDA, gastroduodenal artery; CP, caudate process branch; PP, papillary process branch; RGE, right gastroepiploic artery; CPD, cranial pancreatic duodenal artery; RML, right medial lobe; QL, quadrate lobe; LLL, left lateral lobe; LML, left medial lobe artery. firmed with a measurement of the attenuation values of hepatic parenchyma, which were $72.89 \pm 11.11,76.92 \pm 9.71$, and $128.61 \pm 12.54 \mathrm{HU}$ on the pre-contrast, arterial phase, and portal phase images, respectively.

The celiac artery was identified easily as the first branch of the abdominal aorta with a mean diameter of $5.1 \mathrm{~mm}$ (SD: $0.53)$. The celiac artery coursed cranially and separated into three branches; hepatic, left gastric and splenic arteries. The $4.05 \mathrm{~mm}$ (SD: 0.46), and the right lateral branch was the first branch that arose from the hepatic artery at the dorsal aspect of the main portal vein (Fig. 1). The hepatic artery coursed cranio-ventromedially around the portal vein, and gave off the left branch, which ran towards the left part of the liver. As soon as the left branch departed from the hepatic artery, a relatively smaller artery, the right medial branch, ran toward the right medial and quadrate lobes. On the transverse image at the level of porta hepatis, the right medial branch and left branch were observed at the ventral aspect of the main portal vein (Fig. 1). The course and ramification of the hepatic artery were well visualized on the 3D-volume rendering images (Fig. 2 ). In one dog of this study, the first branch of the hepatic artery was the caudate process artery, which coursed dorsally and supplied the caudate process of the right lateral lobe. The right lateral branch arose from the hepatic artery with right medial and left branches (Fig. 3).

\section{Discussion}

In this study, the arterial phase liver images were sepahepatic artery kept going cranially with a mean size of 
rated successfully from the portal phase image with the fixed-scan method. The early phase of CT, diffuse contrast enhanced liver parenchyma after contrast media injection was noted due to the slow scan time and contrast media injection speed. The development of CT technology made tri- or dual-phased helical liver images possible in human and veterinary medicine. On the other hand, it is difficult to obtain pure arterial phase images in small dogs and cats compared to humans because of their rapid heart rate and small body weight $[4,6]$. Multi-phase liver images have been studied in veterinary medicine. In a study of portal and hepatic angiography in dogs, the arterial phase was obtained at 9.9$20.1 \mathrm{sec}$ and minimum delay time between the arterial and portal phase was $9.5 \mathrm{sec}$ from a dynamic scan with $185 \mathrm{mg}$ iodine $/ \mathrm{kg}$ and $5 \mathrm{ml} / \mathrm{s}$ injection speed [7]. In the present study, a dynamic scan was not performed, but a fixed-scan method was used with 6-7 sec after the injection of contrast medium. A dynamic scan with a small amount of contrast medium could not reflect completely the time attenuation curve of the main scan with different doses of contrast media. Therefore, if the circulatory system is within the normal status, a fixed scan delay system can make consistent images in dogs.

The anatomy of the hepatic arteries is very important for surgical planning for safe removal of the affected parts of the liver in dogs and human patients. Therefore, several anatomic and imaging studies for the hepatic arteries have been performed in dogs [4-7]. Schmidt et al. [4] identified three major types of the hepatic artery branching pattern in dogs, which were the single hepatic artery trunk, two separate branches (most common type) and three or more branches arising directly from the hepatic artery. Other variations were found in the anatomic study of 20 normal dogs with corrosion cast [7]. In half of those dogs, an artery that originated directly from the hepatic artery and supplied the caudate process was found. That artery was the first and the most dorsal branch of the hepatic artery. In the present study, one of seven dogs had similar variants. The caudate process had the first branch of the hepatic artery. Ursic et al. [5] suggested that the artery should be called the caudate process branch (ramus processus caudate) to avoid misinterpretation with the caudate lobe artery, which is supposed to originate from the right lateral branch. Although only one dog had the caudate lobe artery branched from the hepatic artery, further study on a large number of dogs will be needed to determine the proportion of caudate lobe artery variants.

Imaging studies using CT angiography are a relatively noninvasive, simple, and in-vivo method for visualizing the hepatic vasculature in both humans and animals. A dualphase CT angiographic study with a dynamic scan showed good visualization of the hepatic vein, portal vein and hepatic arteries [7]. In that study, they reported that the first branch of the hepatic artery was the right lateral branch and the second was the gastroduodenal artery in two dogs. In the remaining three dogs, the hepatic artery produced the gastroduodenal artery as the first branch [7]. Their results were very differ- ent from the results of previous anatomic studies and the present study. This is presumably because they analyzed only two-dimensional images. The hepatic artery coursed cranially and gave off their branches and ran caudally to form the gastroduodenal artery. Therefore, only 2D-image evaluation could cause a misinterpretation of their ramification patterns. $3 \mathrm{D}$-volume rendering image analysis with multiplanar reconstruction images or navigation system from software should be required for a more accurate evaluation of the hepatic vasculature on CT angiographic images.

The limitation in this study was that only a small number of beagle dogs were evaluated, which could result in fewer anatomic variations due to the lack of breed diversity. Further study will be needed with different breeds or variable sized dogs to make an accurate evaluation of the vascular variation prevalence in dogs.

In conclusion, the arterial phase images for hepatic arterial evaluation are obtained easily with multi-detected CT. Hepatic arterial ramification can be visualized on arterial phase images and 3D-volume rendering analysis techniques. Most dogs had the right lateral branch, left branch, and right medial branch as the main branches of the hepatic artery. Only one dog in this study showed a variant pattern. The variation of the vascular anatomy could be very important for the planning of hepatic lobectomy or vascular-based catheterization procedures in veterinary medicine.

\section{Acknowledgements}

This work was supported by research funds of Chungnam National University.

\section{References}

1. Hiatt JR, Gabbay J, Busuttil RW. Surgical anatomy of the hepatic arteries in 1000 cases. Ann Surg 1994;220:50-52.

2. Onashvili N, Kutateladze M, Tugushi N, Avazashvili I, Mizandari M. Hepatic arterial anatomy variations in patients with hepatocellular carcinoma evaluated by computed tomography angiography. Georgian Med News 2016;216:31-36.

3. Evans HE, De Lahunta A. The heart and arteries. In: Miller's Anatomy of the Dog. 4th ed. pp. 478-487. St. Louis: Elsevier, 2013.

4. Schmidt S, Lohse CL, Suter PF. Branching patterns of the hepatic artery in the dog: arteriographic and anatomic study. Am J Vet Res 1980;41:1090-1097.

5. Ursic M, Ravnik D, Hribernik M, Pecar J, Butinar J, Fazarinc G. Gross anatomy of the portal vein and hepatic artery ramifications in dogs: corrosion cast study. Anat Histol Embryol 2007;36:83-87.

6. Oishi Y, Tani K, Nakazawa H, Itamoto K, Haraguchi T, Taura Y. Anatomical evaluation of hepatic vascular system in healthy beagles using X-ray contrast computed tomography. J Vet Med Sci 2015;77:925-929.

7. Zwingenberger AL, Schwarz T. Dual-phase CT angiography of the normal canine portal and hepatic vasculature. Vet Radiol Ultrasound 2004;45:117-124. 